The initial stage of high intensity pulsed ion beam irradiated magnesium alloys was studied by $\mathrm{MD}$ simulation. Specimens containing $\mathrm{Mg}_{17} \mathrm{Al}_{12}$ precipitation were modeled to investigate the evolution of magnesium alloys during several picoseconds after a high-energy ion impacting. It was found that the $\mathrm{Mg}_{17} \mathrm{Al}_{12}$ precipitation has little effects on the kinetic energy evolution in the heat zone, but considerable effects on strength of kinetic energy peak moving to the deep matrix and on the surface morphology of the magnesium alloy at thermal equilibrium state. The thickness of the heat zone is independent on the temperature of surface region.

Key words: Magnesium alloys; high-intensity pulsed ion beam; $\operatorname{Mg}_{17} \mathrm{Al}_{12}$ precipitation; MD simulation

Magnesium alloys have attracted more and more interests for their high specific strength. However, their outdoor applications are limited due to the low wear and corrosion resistance of the raw materials. Benefit to the surface modification technology, magnesium alloys with modified surface exhibit considerable improvement in wear and corrosion resistance [1-3]. High intensity pulsed ion beam (HIPIB) is a unique technology for magnesium alloys surface modification, which induces a combined improvement in wear and corrosion resistance [1]. The HIPIB technology avoids the problems and technological limitations such as limited adhesive properties [4], non-uniform deformation and residual stress [2, 3], and shallow modified range [5-7]. It is a clean environmental friendship technology with rapid processing rate [8]. During HIPIB irradiation, high-intensity ions with hundreds of $\mathrm{keV}$ energy impact the materials surface [1]. In such energy range, effects of electronic stopping and the electron-phonon interactions will cause the lattice temperature higher than $10^{7} \mathrm{~K}$ in the first $0.3 \mathrm{ps}$ and down to $10^{4} \mathrm{~K}$ at about 6 ps according to the simulation by Zarkadoula et al [9]. Such high lattice temperature will produce ablation plasma with large velocity. The relationship between the kinetic energy and the temperature is 
where $E$ is the total kinetic energy of the ablation plasma containing $n$ ions with mass of $m . k$ is the Boltzmann constant, and $v$ is the velocity of the ions. Supposing the ablation plasma was in local thermal equilibrium with the lattice at the surface region, the lattice with temperature of $10^{4} \mathrm{~K}$ would induced the ablation plasma with velocity of $2.3 \mathrm{~km} / \mathrm{s}$. According to the numerical results of Buttapeng [10] and the experimental data obtained by Yatsui et al.[11-13], the velocity of ablation plasma caused by HIPIB was in the range of 0.7 to $15 \mathrm{~km} / \mathrm{s}$ which is consistent with the results of electronic stopping and the electron-phonon interactions theory. For HIPIB irradiated on Mg alloys, the high lattice temperature in the initial several picoseconds will intensively change the state of irradiated magnesium alloys surface and considerably affect the following evolution. The experimental investigation on this short processing is seriously restricted by the extremely rapid processing speed. For the high spatial resolution, the molecular dynamics (MD) simulations have been extensively used to investigate the high energy density deposition process in an ultrashort time at the atomic level such as laser-material interactions [14-16]. In this paper, MD simulations of the initial stage of the HIPIB irradiation on the magnesium alloy were performed. The work focus on the materials behavior induced by an ion impacting event (IIE). The computational model and simulation procedures for the HIPIB irradiation of magnesium alloys at the atomic level are presented in the following.

The simulation boxes were constructed with dimension of $6.419 \mathrm{~nm}, 6.671 \mathrm{~nm}$, and $30 \mathrm{~nm}$ in $\mathrm{X}, \mathrm{Y}$, and $\mathrm{Z}$ directions, respectively. Pure Mg crystal was prepared by filling the simulation box from 0 to $26.153 \mathrm{~nm}$ along $\mathrm{Z}$ direction in an h.c.p lattice with constant of $0.3209 \mathrm{~nm}$ as shown in Fig. 1 (a). Samples with $\mathrm{Mg}_{17} \mathrm{Al}_{12}$ precipitation were prepared by substitute a cubic block region with length of $3.165 \mathrm{~nm}$ of pure $\mathrm{Mg}$ crystal at various depths by $\mathrm{Mg}_{17} \mathrm{Al}_{12}$ precipitation in Fig 1. (b) to (f). Fig. $1(\mathrm{~g})$ shows the structure of $\mathrm{Mg}_{17} \mathrm{Al}_{12}$ precipitation. It contains 1566 atoms and has an $\alpha$-Mn-type cubic unit cell (space group I43m) with lattice parameter of $1.056 \mathrm{~nm}$ [17]. The MD samples depicted in Fig.1(a) (f) were named A to F. For samples B to F, the distance between the top interface of the $\mathrm{Mg}_{17} \mathrm{Al}_{12}$ precipitation and top surface of the magnesium matrix are $0,1.5,3.0,4.5$ and $16.5 \mathrm{~nm}$, respectively. The mass center of the $\mathrm{Mg}_{17} \mathrm{Al}_{12}$ precipitation were at $\mathrm{X}=3.209 \mathrm{~nm}, \mathrm{Y}=3.336 \mathrm{~nm}$ for all magnesium samples. Periodic boundary conditions were imposed in $\mathrm{X}$ and $\mathrm{Y}$ directions. The free boundary condition in $\mathrm{Z}$ direction was used to avoid the reflection effect caused by using fixed or period boundary conditions. The rigid motion of the specimen was restricted by the bottom surface atoms through setting the force zero. The atomic interactions in the magnesium alloy specimen were described by Finnis-Sinclair embedded-atom method ( potential \#4 in Ref [18]). Because $\mathrm{Mg}_{17} \mathrm{Al}_{12}$ phase has a different lattice structure with the magnesium matrix, artificial high local stress could appear near the interface of the precipitation and the magnesium matrix. To eliminate the artificial high local stress of the samples with $\mathrm{Mg}_{17} \mathrm{Al}_{12}$ precipitation, the Polak-Ribiere version of the conjugate gradient (CG) algorithm was employed to 
carry out an energy minimizing procedure for samples B to F. Then, Samples A to F were relaxed at $300 \mathrm{~K}$ and 0 bar for $15 \mathrm{ps}$ within isothermal-isobaric NPT ensemble (Nose-Hoover thermostat) to its equilibrium configuration. The positions of all atoms in specimen were stored for the following simulations. The lattice temperature $T_{\mathrm{s}}$ of the surface region with thickness of $1.048 \mathrm{~nm}$ were assigned a value in the range of $2 \times 10^{4}$ to $1.8 \times 10^{5} \mathrm{~K}$ at $0 \mathrm{ps}$ to simulate the effect of IEE. The simulations of HIPIB irradiation procedure were achieved within microcanonical NVE ensemble for $3 \mathrm{~ns}$ which is long enough in our simulation. OVITO code [19] was used to visualize and analysis the simulation results. All the MD simulations were performed by using the Lammps (Large-scale Atomic/Molecular Massively Parallel Simulator) software [20].

The evolution of the atoms in samples $A$ to $F$ during the initial several picoseconds with $T_{\mathrm{s}}$ in the range of $2 \times 10^{4}$ to $1.8 \times 10^{5} \mathrm{~K}$ are obtained by the MD simulation. According to the simulation results, the evolution of kinetic energy in the different samples show little difference with the same $T_{\mathrm{s}}$. For clarity, only results for sample A and B are given. Fig. 2 shows the kinetic energy maps of pure $\mathrm{Mg}$ sample A and magnesium alloys sample $\mathrm{B}$ for $T_{\mathrm{s}}=6 \times 10^{4} \mathrm{~K}$ in the first $2.5 \mathrm{ps}$, respectively. The atoms with kinetic energy less than $0.119 \mathrm{eV}$ (corresponding to the boiling point of $\mathrm{Mg} 1107{ }^{\circ} \mathrm{C}$. For convenience, it will be named $E_{\mathrm{b}}$ in the following) are deleted to reveal the form of kinetic energy evolution. The IIE brings huge kinetic energy which localize to the surface region and produce a heat zone on the surface. A remarkable result obtained from the simulation is that a moving plane was created with average velocity $v_{\mathrm{p}}$ from the heat zone during first $0.5 \mathrm{ps}$. As shown in Fig. 2, the heat zone thickness and the $v_{\mathrm{p}}$ are almost the same for both sample A and B. The change of the heat zone thickness during the first $2.5 \mathrm{ps}$ are not observed. According to the simulation results, the $v_{\mathrm{p}}$ is about $9 \mathrm{~km} / \mathrm{s}$.

Fig. 3 (a-f) show the per-atom kinetic energy averaged over 0.1 ps in 29 bins from 0 to $300 \mathrm{~nm}$ for sample A to F, respectively (bins centered at 0 and $30 \mathrm{~nm}$ were not calculated). The bin 1 is the first bin which centered at $1 \mathrm{~nm}$ with boundary of 0.5 $\mathrm{nm}$ and $1.5 \mathrm{~nm}$. As can be observed, the kinetic energy plots in range of bin 20 to bin 30 increase dramatically during the initio $2.5 \mathrm{ps}$, and decrease with time and depth. Since the surface is in the $26^{\text {th }}$ bin, it can be deduced that the thickness of the heat zone was $6 \mathrm{~nm}$ which was the length of 6 bins. The max value of kinetic energy in the heat zone is nearly $9 \mathrm{eV}$ in the initial state and drop to about $1 \mathrm{eV}$ after $2.5 \mathrm{ps}$. Such huge loss of the kinetic energy in the heat zone should be caused by ablation process, because no considerable thermal conduction process is observed. The kinetic energy plots for sample A to $\mathrm{F}$ at the same time are nearly the same in this range of bins. It implies that the position of the $\mathrm{Mg}_{17} \mathrm{Al}_{12}$ precipitation has little effects on the kinetic energy evolution in the heat zone. The kinetic energy plots in the other bins show moving peaks with value near $E_{\mathrm{b}}$ moved from the heat zone to the matrix in a constant velocity of about $9 \mathrm{~km} / \mathrm{s}$. It was consistent with behavior of the move plane in the kinetic energy maps in Fig.2. The value of the constant velocity equaled to $v_{\mathrm{p}}$. It suggested that a small part of kinetic energy can transformed from the heat zone to the deep matrix in shock wave style in the HIPIB process. The strength of kinetic peak for pure $\mathrm{Mg}$ was larger than magnesium alloys. The latter decreased from 0.13 to $0.08 \mathrm{eV}$ 
while the depth of the $\mathrm{Mg}_{17} \mathrm{Al}_{12}$ precipitation increasing. It implied the position of the $\mathrm{Mg}_{17} \mathrm{Al}_{12}$ precipitation has considerable effects on strength of kinetic energy peak. Fig. 4 gives the plots of (a) temperature, (b) potential energy, and (c) number of atoms in the simulation box vs time. The temperature is defined as the average kinetic energy of the atoms in the simulation box during $0.1 \mathrm{ps}$. The temperature plots are the same for all samples. It demonstrated the position of $\mathrm{Mg}_{17} \mathrm{Al}_{12}$ precipitation has little effect on the thermal conduction process. Although the plots of potential energy for pure $\mathrm{Mg}$ were higher from the ones for magnesium alloys, the shape of the plots were almost the same. It means the conversion rate for kinetic energy to potential energy was independent on the position of the $\mathrm{Mg}_{17} \mathrm{Al}_{12}$ precipitation. The ablation process was also independent on the position of the $\mathrm{Mg}_{17} \mathrm{Al}_{12}$ precipitation since the number of atoms plots between the pure $\mathrm{Mg}$ and magnesium alloys have similar shape. The differences in the potential energy and number of atoms between pure $\mathrm{Mg}$ and magnesium alloy were only caused by the structure difference between $\mathrm{Mg}$ lattice and the $\mathrm{Mg}_{17} \mathrm{Al}_{12}$ precipitation lattice.

Fig.5 (a-d) show the average per-atom kinetic energy averaged over $0.1 \mathrm{ps}$ for sample B with different $T_{\mathrm{S}}$ which is in the rage of $2 \times 10^{4}$ to $1.8 \times 10^{5} \mathrm{~K}$, respectively. The kinetic energy in range of 20 to 30 bins for the case with higher $T_{\mathrm{s}}$ is larger than that with lower $T_{\mathrm{s}}$. However, the thickness of the heat zone was fixed at $6 \mathrm{~nm}$ for all $T_{\mathrm{s}}$. It suggests the value of $T_{\mathrm{s}}$ only affect the kinetic energy of the heat zone, not the thickness. The constant thickness implied that the solid and liquid phase at the interface between the matrix and ablation atoms may act as thermal barrier which prevent the heat introduced by IEE from entering the matrix. The kinetic energy plots in the 0 to 20 bins also show moving peaks from the heat zone to the deep matrix. The velocity of the peak is a constant about $9 \mathrm{~km} / \mathrm{s}$. It was noted that if the value of the energy peak is higher than the boiling point energy $E_{\mathrm{b}}$, it will decrease with time and be stable near $E_{\mathrm{b}}$ finally. Therefore, the defects in the matrix such as vacancy or interstitial atoms may be annihilated during the energy peak passing in the initial stage.

The morphology and structure of the surface is important to the tribology performance of the materials. Fig. 6 shows the surface morphology of the samples A to $\mathrm{F}$ after HIPIB irradiation at 3 ns. The surface of all samples had a wave-like perturbation morphology. Comparing to the sample $\mathrm{E}$, the surface of $\mathrm{A}$ to $\mathrm{D}$ and $\mathrm{F}$ shows larger perturbations feature. Fig. 7 shows the top surface area of the samples A to F. Each point in Fig. 7 represents the average value from 10 simulations. Pure $\mathrm{Mg}$ metal and the magnesium alloy containing an $\mathrm{Mg}_{17} \mathrm{Al}_{12}$ precipitation on the surface have the largest top surface area. As the distance between the top interface of the $\mathrm{Mg}_{17} \mathrm{Al}_{12}$ precipitation and the top surface increasing, the top surface area decreased. Three factors may result in the wave-like perturbation morphology difference: one is the selective ablation. When different kinds of atoms on the surface of materials receives energy from the HIPIB, the ones with lower melting point will depart from the matrix first, i.e. the ablation rates is different for different kinds of atoms, and the remains will from a wave-like perturbation morphology. Defining the ablation rates $\mathrm{R}_{\mathrm{A}}$ 


$$
\mathrm{R}_{\mathrm{A}}=\mathrm{a} / \mathrm{b}
$$

where $\mathrm{a}$ is the number of atoms removed from the simulation box, $\mathrm{b}$ is the number of atom the number of atoms in the initial simulation box. The ablation rates $\mathrm{R}_{\mathrm{A}}$ for samples $\mathrm{A}$ to $\mathrm{F}$ were almost the same, and the difference between the ablation rates were smaller than $1 \%$. as shown in Tab. 1 . Therefore, the effect of the selective ablation for different samples on the surface roughness could be neglected. The other is the deformation of the material responding to the heat. The crystal structure of the materials such as lattice type, lattice length etc., would be changed in the heating process and kept in the consequent extremely rapid cooling process. The crystal structure changing will produce a stress field which has long-range interaction with the surface region and affect the surface roughness. To investigate the contribution of this factor, the radial distribution function was calculated. The detailed coordination analysis showned that the posted irradiation surface region had the similar radial distribution function to the bulk. Similar radial distribution function demonstrated the atoms in the surface region keep the same crystal structure as the bulk. It suggests that the crystal structure of magnesium alloy is stable in the rapid heating process such as HIPIB and the structure stability lead to the surface region remains the same structure of bulk. The calculation results are consistent with the experimental ones which show clear crystalline XRD patterns [1,21]. Therefore, the effect of the crystal structure changing on the surface morphology could also be neglected. The third one is the atom diffusion under HIPIB irradiation. The atoms on the surface jump from one equilibrium position to another one in the system with high temperature, and have considerable effect on the morphology of the surface. According a serious snapshot of the system, atoms in the surface region of sample A and B jump more frequently than the others. This variation of diffusion ability of atoms in different samples may originate from the difference of the time-dependent force field induced by thermal shock wave formed in the sudden heating of the surface. The pure $\mathrm{Mg}$

Additionally, Fig. 6 and Fig. 7 suggest the surface roughness of pure Mg matrix was larger than the $\mathrm{Mg}$ - $\mathrm{Al}$ alloy. As the concentration of $\mathrm{Al}$ increasing, the content of $\mathrm{Mg}_{17} \mathrm{Al}_{12}$ precipitation in the magnesium matrix will increase. Therefore, the magnesium alloy with higher Al concentration should have smoother surface than the one with lower $\mathrm{Al}$ concentration.

Fig. 8 shows the equilibrium structure of the $\mathrm{Mg}_{17} \mathrm{Al}_{12}$ precipitation after HIPIB irradiation. After the HIPIB irradiation, the shapes of cubic $\mathrm{Mg}_{17} \mathrm{Al}_{12}$ precipitation changed into an irregular shape. The modification of the $\mathrm{Mg}_{17} \mathrm{Al}_{12}$ precipitation explains the surface wear resistance increased after the HIPIB irradiation. Compared with most precipitation-hardenable aluminium alloys, magnesium alloys shows poor precipitation hardening. Most $\mathrm{Mg}_{17} \mathrm{Al}_{12}$ precipitates lie on the basal plane and are not effective obstacles to the motion of dislocations[17, 22]. After the HIPIB irradiation, the modified $\mathrm{Mg}_{17} \mathrm{Al}_{12}$ precipitates will penetrate the basal plane and formed into obstacle to the motion of dislocations. The modification of the $\mathrm{Mg}_{17} \mathrm{Al}_{12}$ precipitation can be quantity by the mean standard err (MSE) of displacement of the atoms. Fig. 9 shows the MSE of displacement for the atoms in $\mathrm{Mg}_{17} \mathrm{Al}_{12}$ precipitation and the 
[1] P. Li, X.G. Han, J.P. Xin, X.P. Zhu, M.K. Lei, Wear and corrosion resistance of AZ31 magnesium alloy irradiated by high-intensity pulsed ion beam, Nucl. Instrum. Methods Phys. Res., Sect. B 266 (2008) 3945-3952.

[2] G. Abbas, L. Li, U. Ghazanfar, Z. Liu, Effect of high power diode laser surface melting on wear resistance of magnesium alloys, Wear, 260 (2006) 175.

[3] S. Ignat, P. Sallamand, D. Grevey, M. Lambertin, Magnesium alloys laser (Nd:YAG) cladding and alloying with side injection of aluminium powder, Appl. Surf. Sci. , 225 (2004) 124-134.

[4] H. Hoche, H. Scheerer, D. Probst, E. Broszeit, C. Berger, Development of a plasma surface treatment for magnesium alloys to ensure sufficient wear and corrosion resistance, Surf. Coat. Technol. , 174 (2003) 1018-1023.

[5] H.X. Liu, Q. Xu, D.M. Xiong, B. Lin, C.L. Meng, Microstructure and corrosion resistance of AZ91D magnesium alloy treated by hybrid ion implantation and heat treatment, Vacuum, 89 (2013) 233-237.

[6] I. Nakatsugawa, R. Martin, E.J. Knystautas, Improving corrosion resistance of AZ91D magnesium alloy by nitrogen ion implantation, Corrosion, 52 (1996) 921-926.

[7] M. Vilarigues, L.C. Alves, I.D. Nogueira, N. Franco, A.D. Sequeira, R.C. daSilva, Characterisation of corrosion products in Cr implanted Mg surfaces, Surf. Coat. Technol. , 158-159 (2002) 328-333.

[8] V.A. Shulov, A.S. Novikov, A.G. Paikin, A.B. Belov, A.F. Lvov, G.E. Remnev, Crater formation on the surface of refractory alloys during high-power ion-beam processing, Surf. Coat. Technol. , 201 (2007) 8654-8658.

[9] E. Zarkadoula, S.L. Daraszewicz, D.M. Duffy, M.A. Seaton, I.T. Todorov, K. Nordlund, M.T. Dove, K. Trachenko, Electronic effects in high-energy radiation damage in iron, J. Phys.: Condens. Matter 26 (2014) 085401.

[10] C. Buttapeng, Numerical analysis of ablation plasma produced by pulsed ion beam for various 
solid targets, Vacuum, 84 (2010) 496-500.

[11] K. Yatsui, H. Shinkai, K. Kashine, W. Jiang, M. Kagihiro, N. Harada, Foil acceleration by intense pulsed ion beam ablation plasma, Jpn. J. Appl. Phys., Part 140 (2001) 955-959.

[12] W. Jiang, T. Sakagami, K. Masugata, K. Yatsui, 3-DIMENSIONAL, TIGHT FOCUSING OF INTENSE PULSED LIGHT-ION BEAM BY SPHERICAL PLASMA-FOCUS DIODE, Laser Part. Beams 13 (1995) 343-350. [13] K. Yatsui, X.D. Kang, T. Sonegawa, T. Matsuoka, K. Masugata, Y. Shimotori, T. Satoh, S. Furuuchi, Y. Ohuchi, T. Takeshita, H. Yamamoto, Applications of intense pulsed ion-beam to materials science, Phys. Plasmas, 1 (1994) 1730-1737.

[14] Z. Lin, E. Leveugle, E.M. Bringa, L.V. Zhigilei, Molecular dynamics simulation of laser melting of nanocrystalline Au, J. Phys. Chem. C 114 (2010) 5686-5699.

[15] D.A. Thomas, Z. Lin, L.V. Zhigilei, E.L. Gurevich, S. Kittel, R. Hergenroder, Atomistic modeling of femtosecond laser-induced melting and atomic mixing in Au film - Cu substrate system, Appl. Surf. Sci. , 255 (2009) 9605-9612.

[16] Z.Q. Li, J. Wang, T. Sun, Atomistic Simulations of Ultrashort Pulsed Laser Ablation of Polycrystalline Diamond, Curr. Nanosci., 9 (2013) 804-811.

[17] R. Zeng, Y. Chiu, I.P. Jones, Characterisation of nano-sized Al-Mn-(Mg) particles in AZ91 and their effect on Mg17Al12 precipitation, J. Alloys Compd. , 579 (2013) 34-38.

[18] M.I. Mendelev, M. Asta, M.J. Rahman, J.J. Hoyt, Development of interatomic potentials appropriate for simulation of solid-liquid interface properties in Al-Mg alloys, Philos. Mag. , 89 (2009) 3269-3285.

[19] A. Stukowski, Computational Analysis Methods in Atomistic Modeling of Crystals, JOM 66 (2014) 399-407.

[20] S. Plimpton, Fast parallel algorithms for short-range molecular dynamics, J. Comput. Phys. , 117 (1995) 1-19.

[21] C. Chena, M. Wang, D. Wang, R. Jin, Y. Liu, Microstructure and corrosion behavior of Mg-Nd coatings on AZ31 magnesium alloy produced by high-energy micro-arc alloying process, J. Alloys Compd. , 438 (2007) 321-326.

[22] S. Celotto, TEM study of continuous precipitation in Mg-9 Wt\%Al-1 Wt\%Zn alloy, Acta Mater. , 48 (2000) 1775-1787. 


\section{Table Caption}

Tab. 1 The ablation rate of different samples

4

5

6

9

10

11

12

13

14

15

16

17

18

19

20

21

22

23

24

25

26

27

28

29

30

31

32

33

34

35

36

37

38

39

40

41

42

43

44

45

46

47

48

49

50

51

52

53

54

55

56

57

58

59

60

61

62

63

64

65 


\section{Figure Captions}

Fig. 1 The MD samples of HIPIB irradiation on the magnesium alloy surface utilized in current study

Fig. 2 The kinetic energy maps of (a-e) pure Mg sample A and (f-j) magnesium alloys sample B for $T_{\mathrm{s}}=6 \times 10^{4} \mathrm{~K}$ in the first $2.5 \mathrm{ps}$.

Fig. 3 The per-atom kinetic energy averaged over 100 time steps in 30 bins span the box in $\mathrm{Z}$ dimension for sample $\mathrm{A}$ to $\mathrm{F}$.

Fig. 4 The plots of (a) temperature, (b) potential energy, and (c) number of atoms in the simulation box vs. time.

Fig. 5 The per-atom kinetic energy averaged over 100 timesteps in 30 bins span the box in $\mathrm{Z}$ dimension for sample $\mathrm{B}$ with different $T_{\mathrm{s}}$ which is in the rage of $2 \times 10^{4}$ to $1.8 \times 10^{5} \mathrm{~K}$.

Fig. 6 The surface morphology of the magnesium alloy samples A to F after HIPIB irradiation at $3 \mathrm{~ns}$.

Fig. 7 The top surface area of the samples A to F.

Fig. 8 The structure of the $\mathrm{Mg}_{17} \mathrm{Al}_{12}$ precipitation after HIPIB irradiation at 3 ns.

Fig. 9 The mean standard err displacement for the atoms in $\mathrm{Mg}_{17} \mathrm{Al}_{12}$ precipitation and the corresponding $\mathrm{Mg}$ atoms in sample $\mathrm{A}$ 
Table

\begin{tabular}{|l|l|l|l|l|l|l|}
\hline Sample & A & B & C & D & E & F \\
\hline Ablation rate $\left(\mathrm{R}_{\mathrm{A}}\right)$ & $5.25 \%$ & $4.62 \%$ & $5.66 \%$ & $4.40 \%$ & $4.45 \%$ & $4.41 \%$ \\
\hline
\end{tabular}


(a)

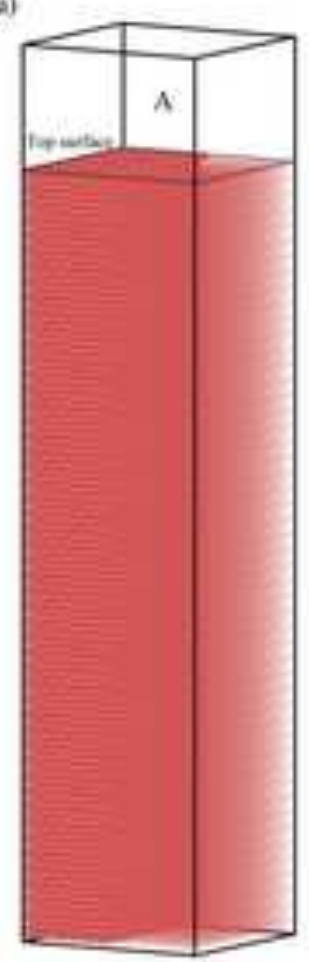

(c)

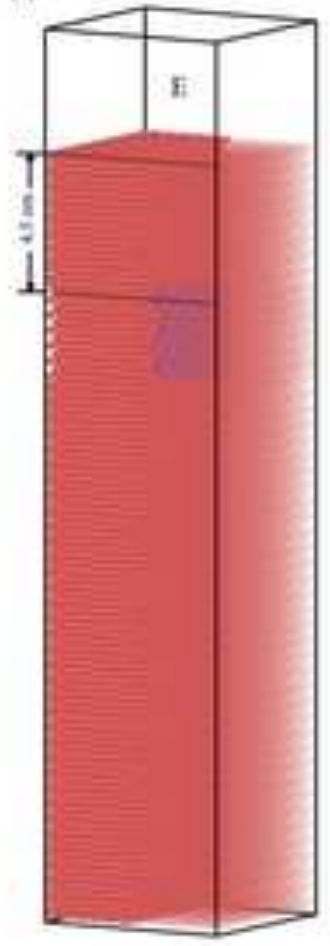

(b)

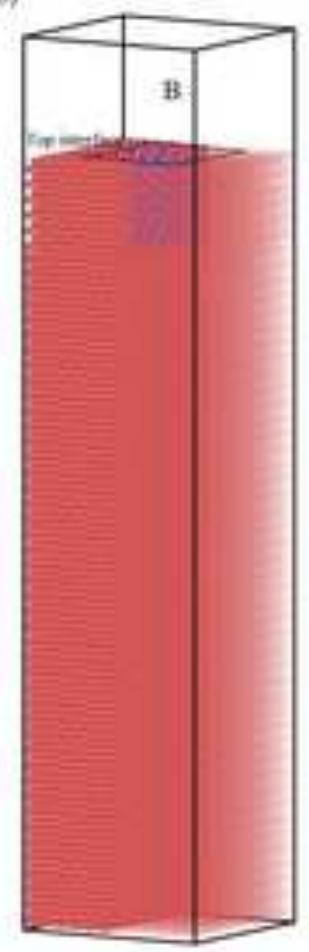

(h)

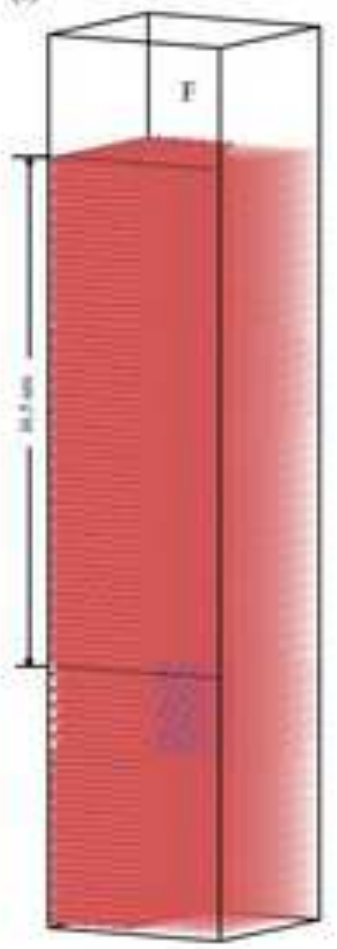

(c)

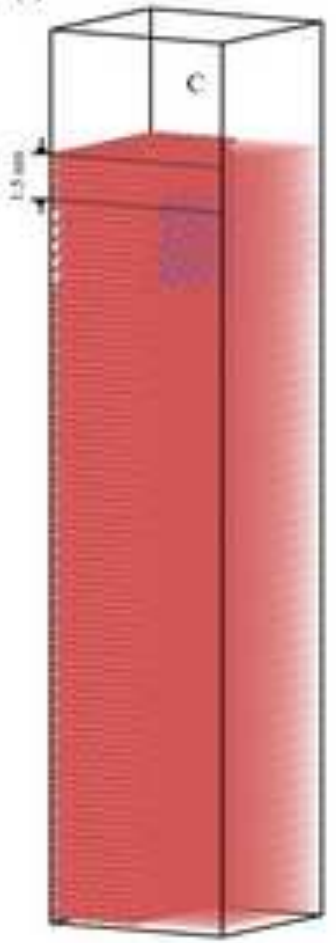

(d)

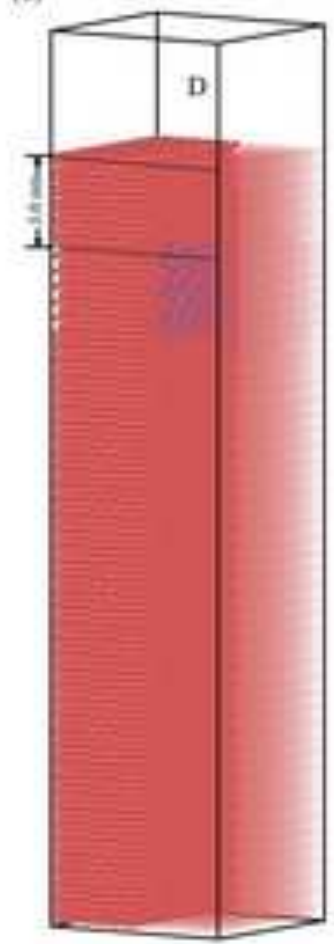

(g)

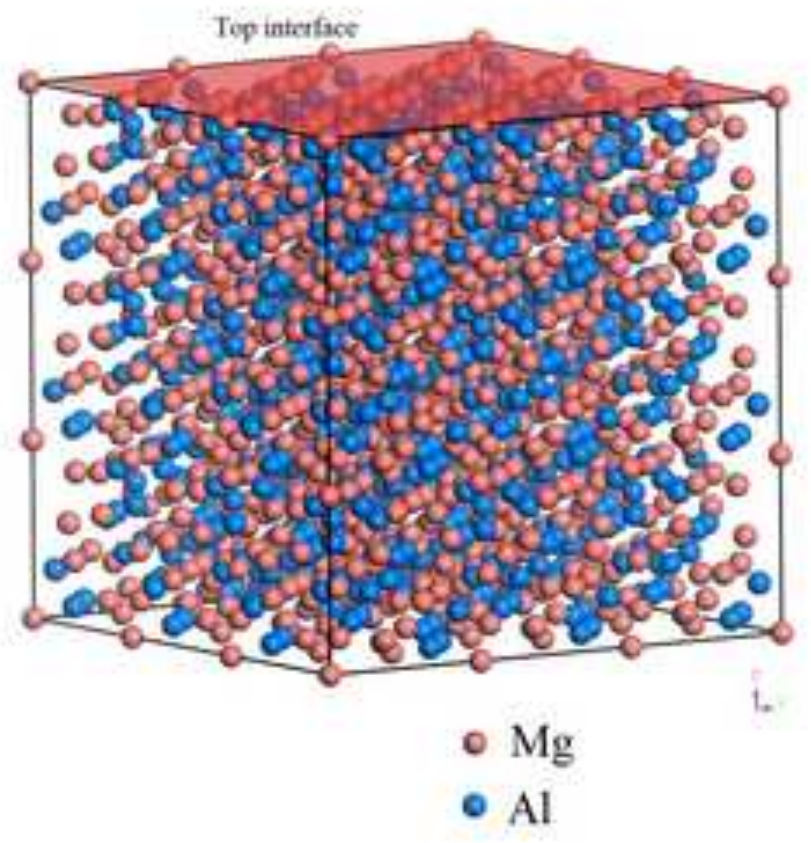



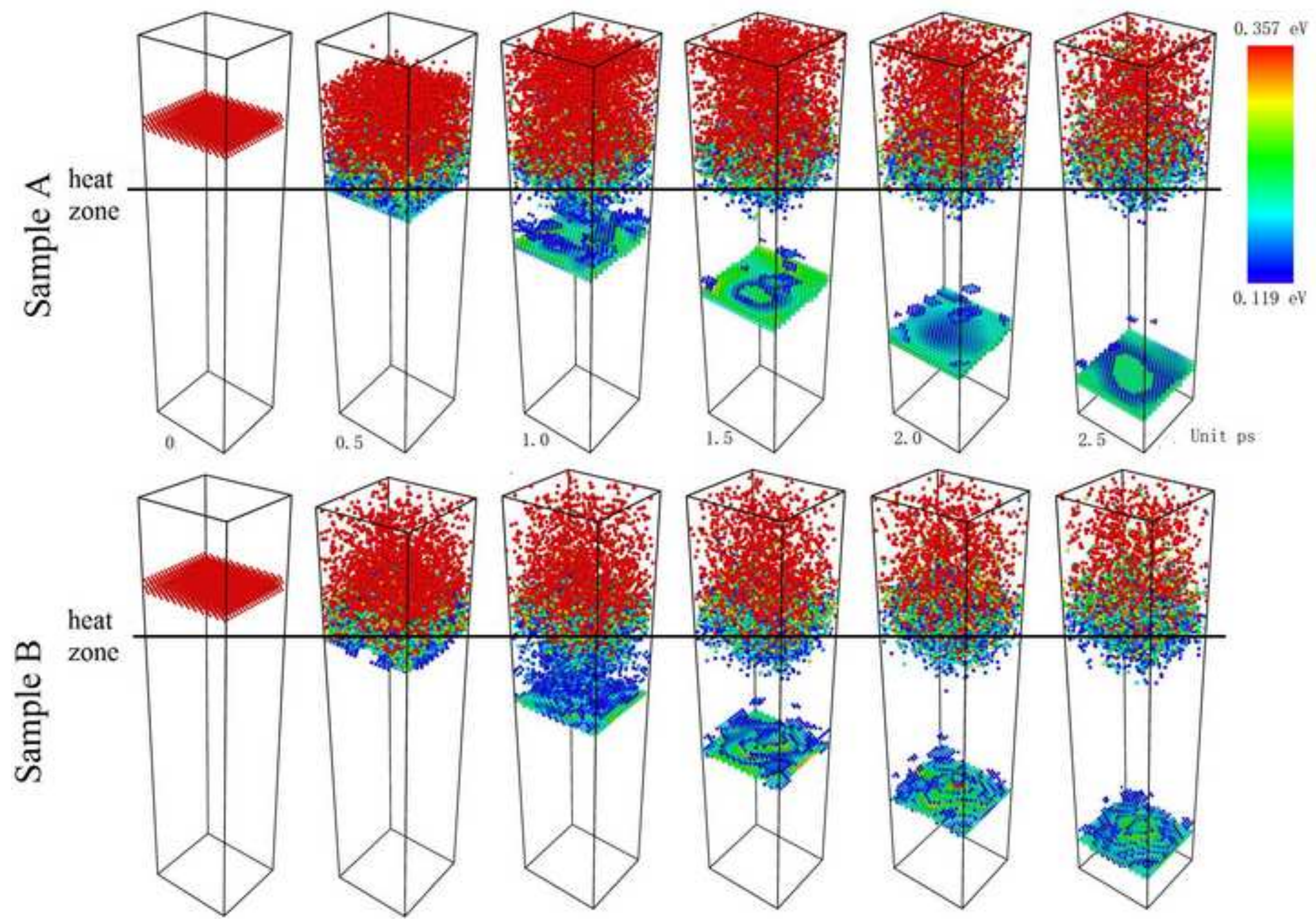

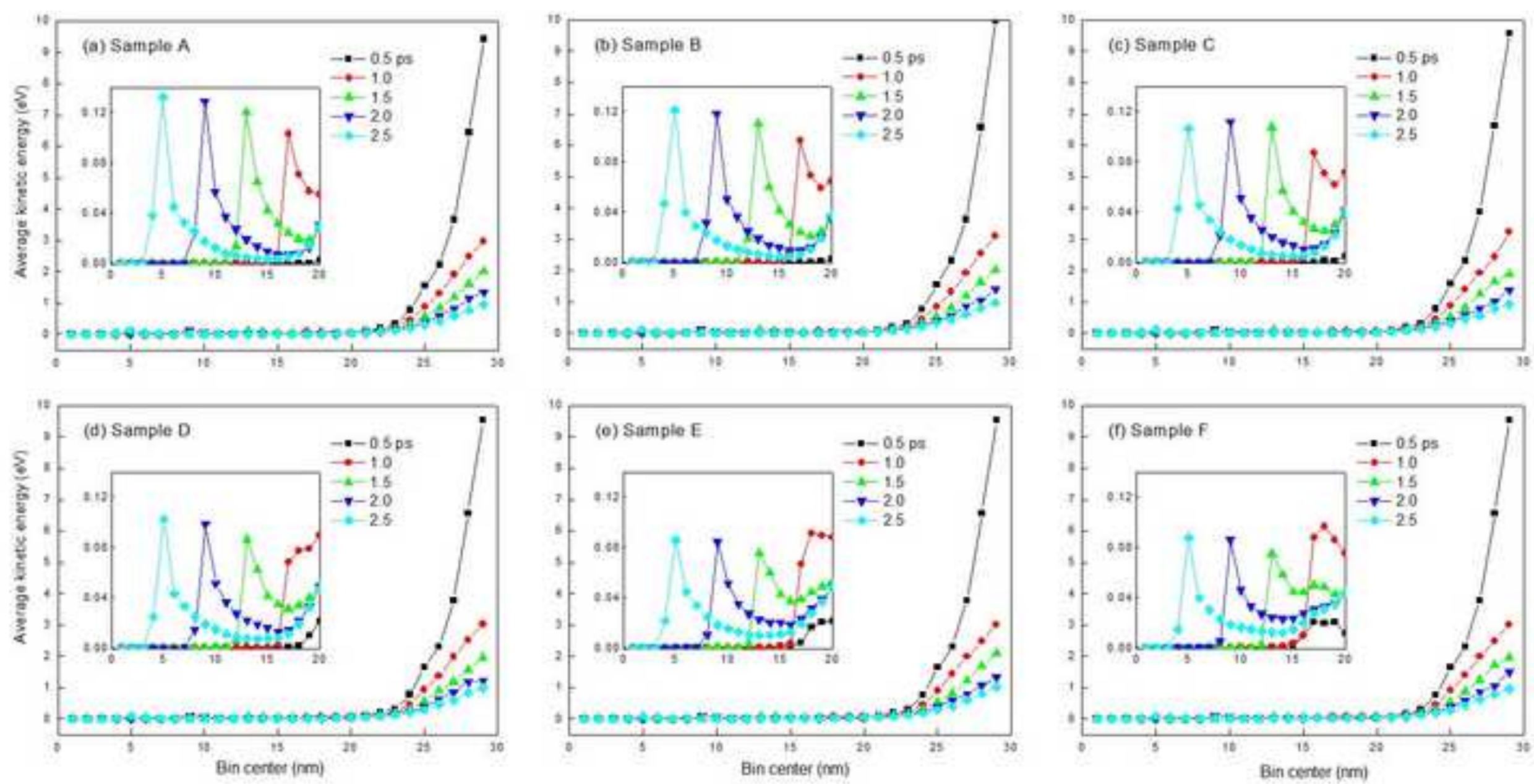
Figure 4
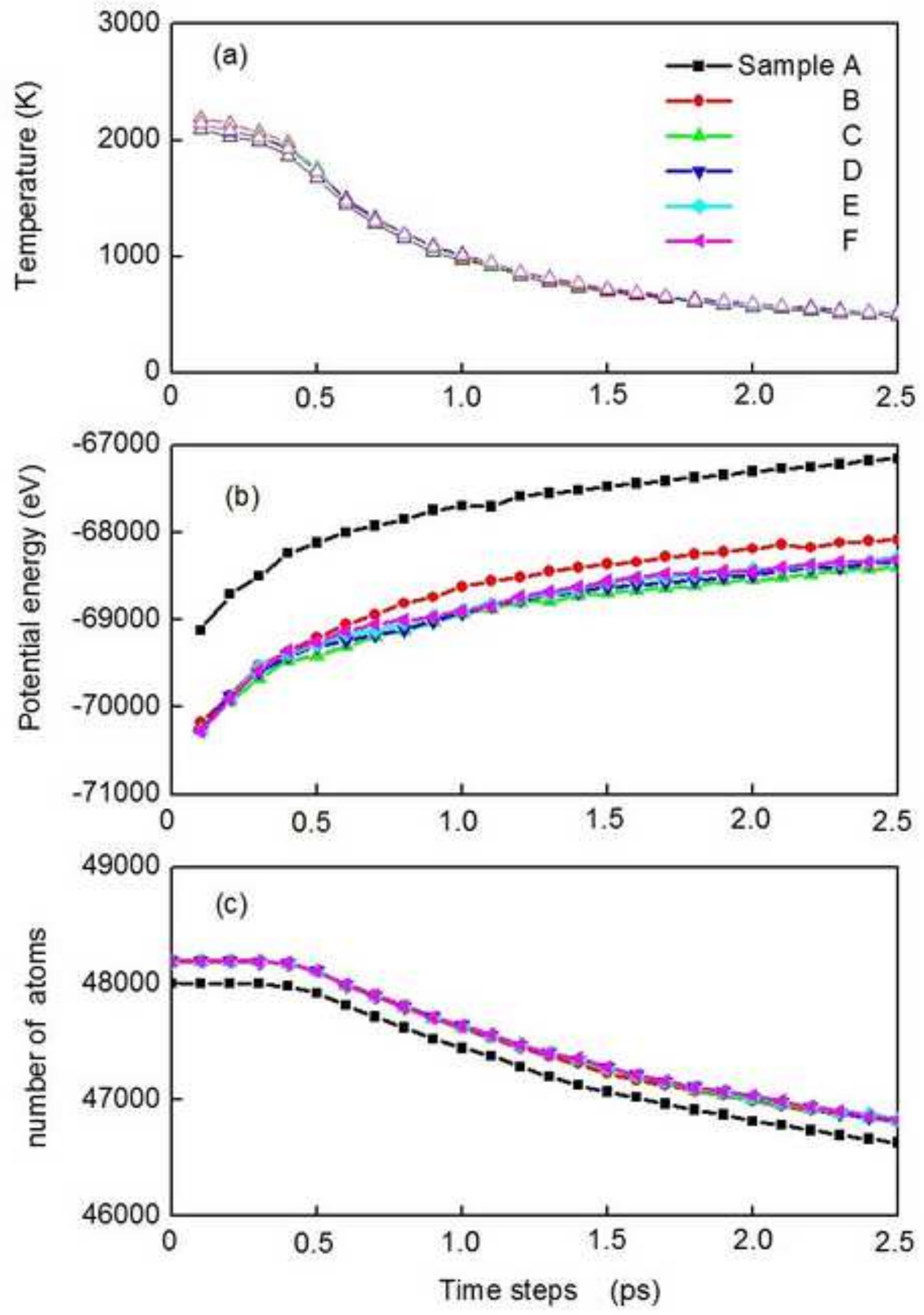

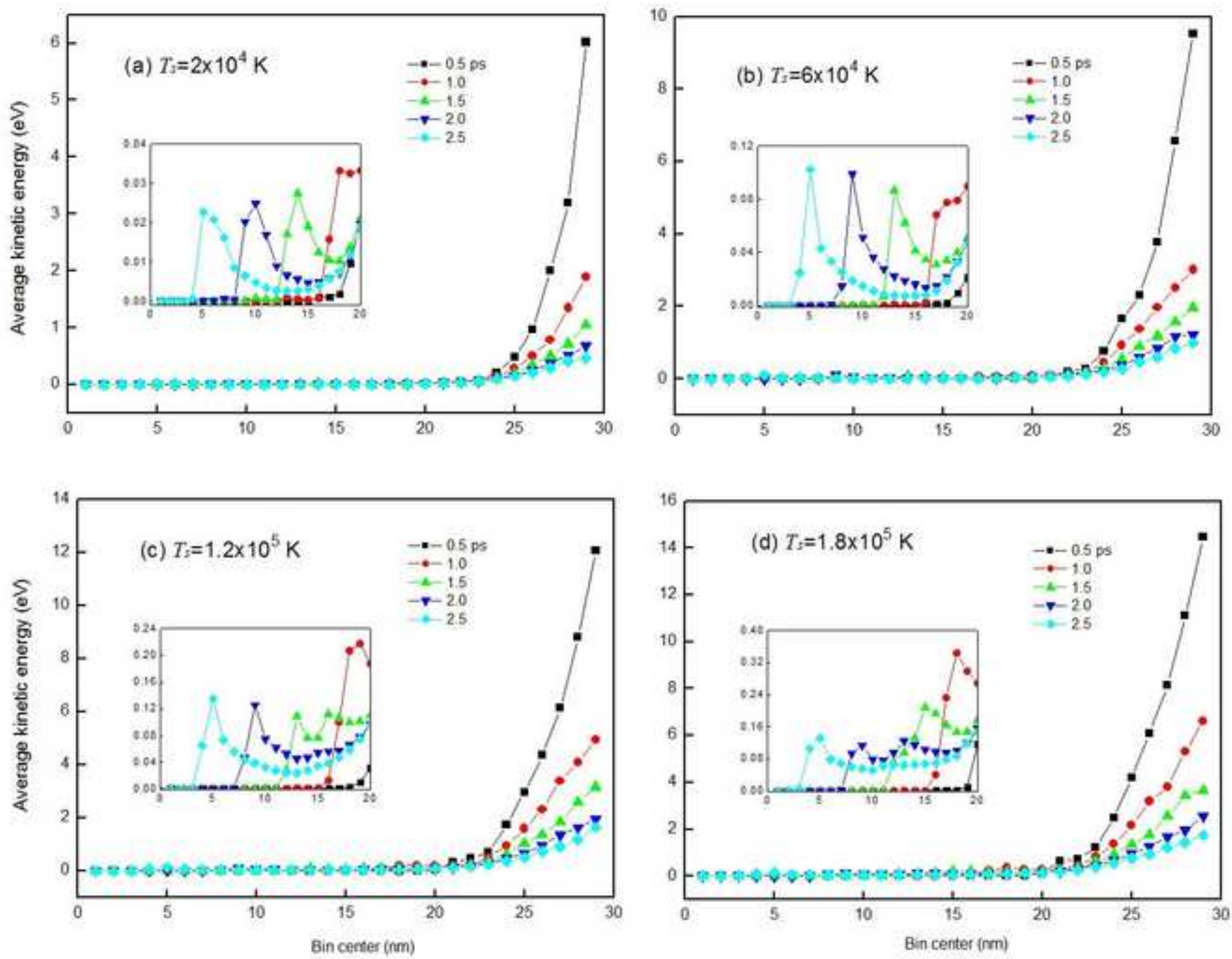
Figure 6

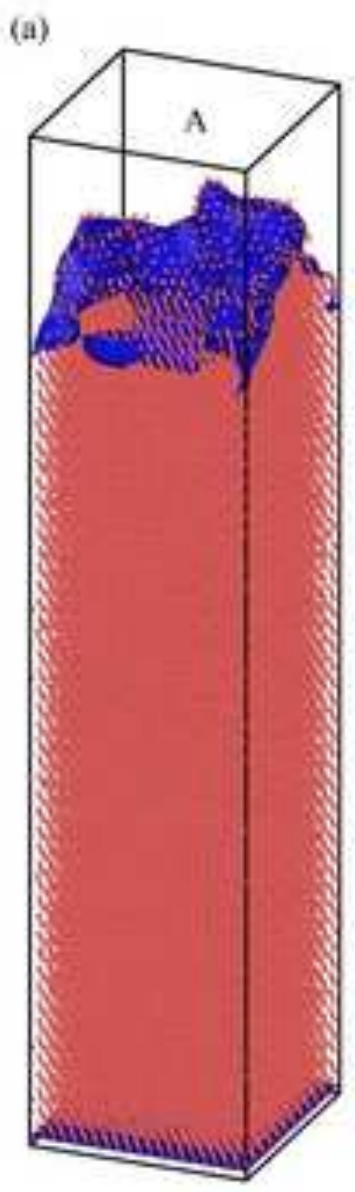

(b)

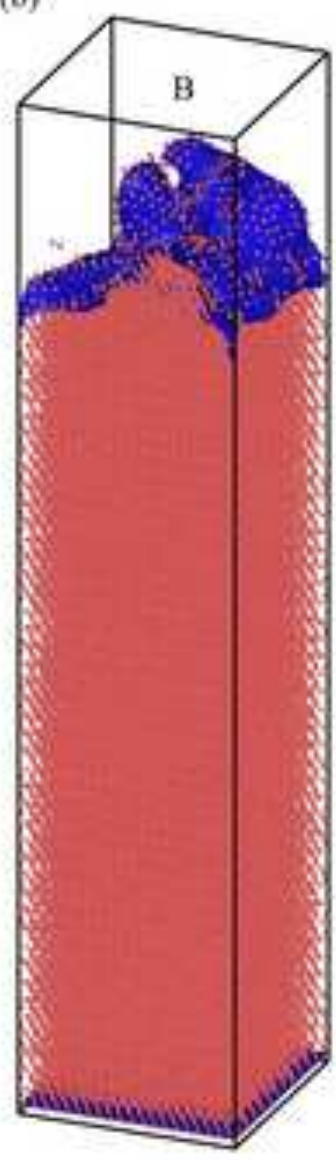

(c)

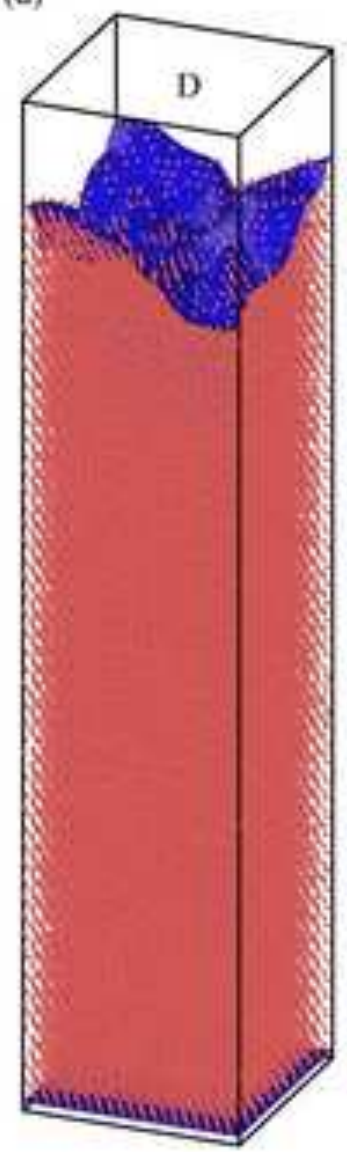

(c)

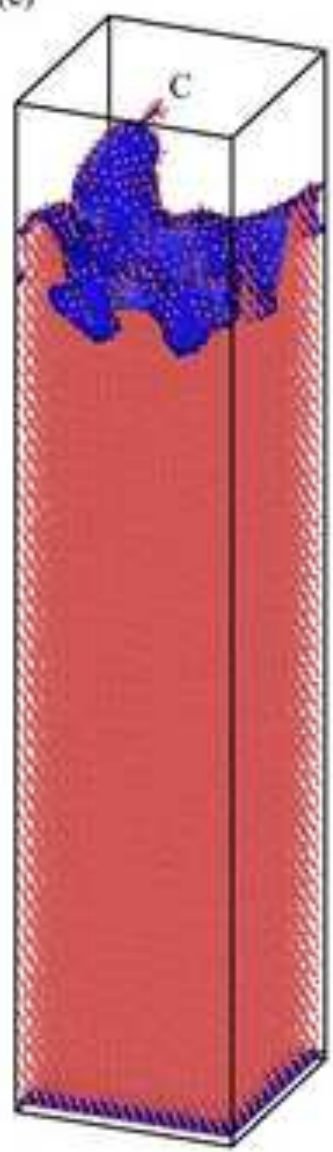

(f)

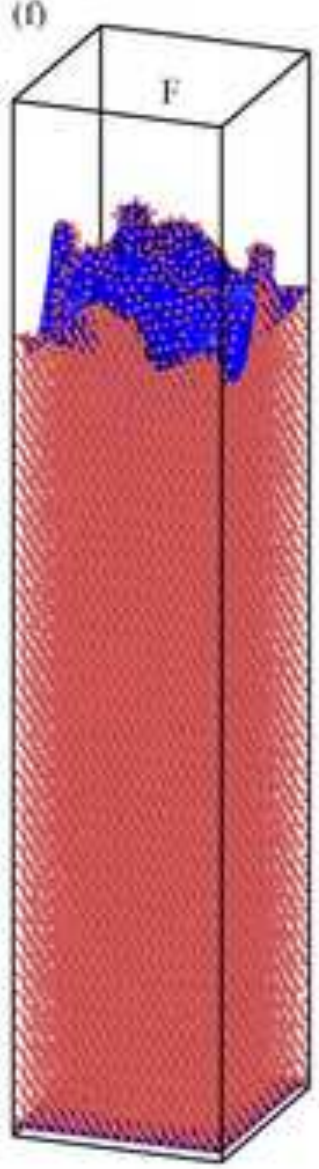




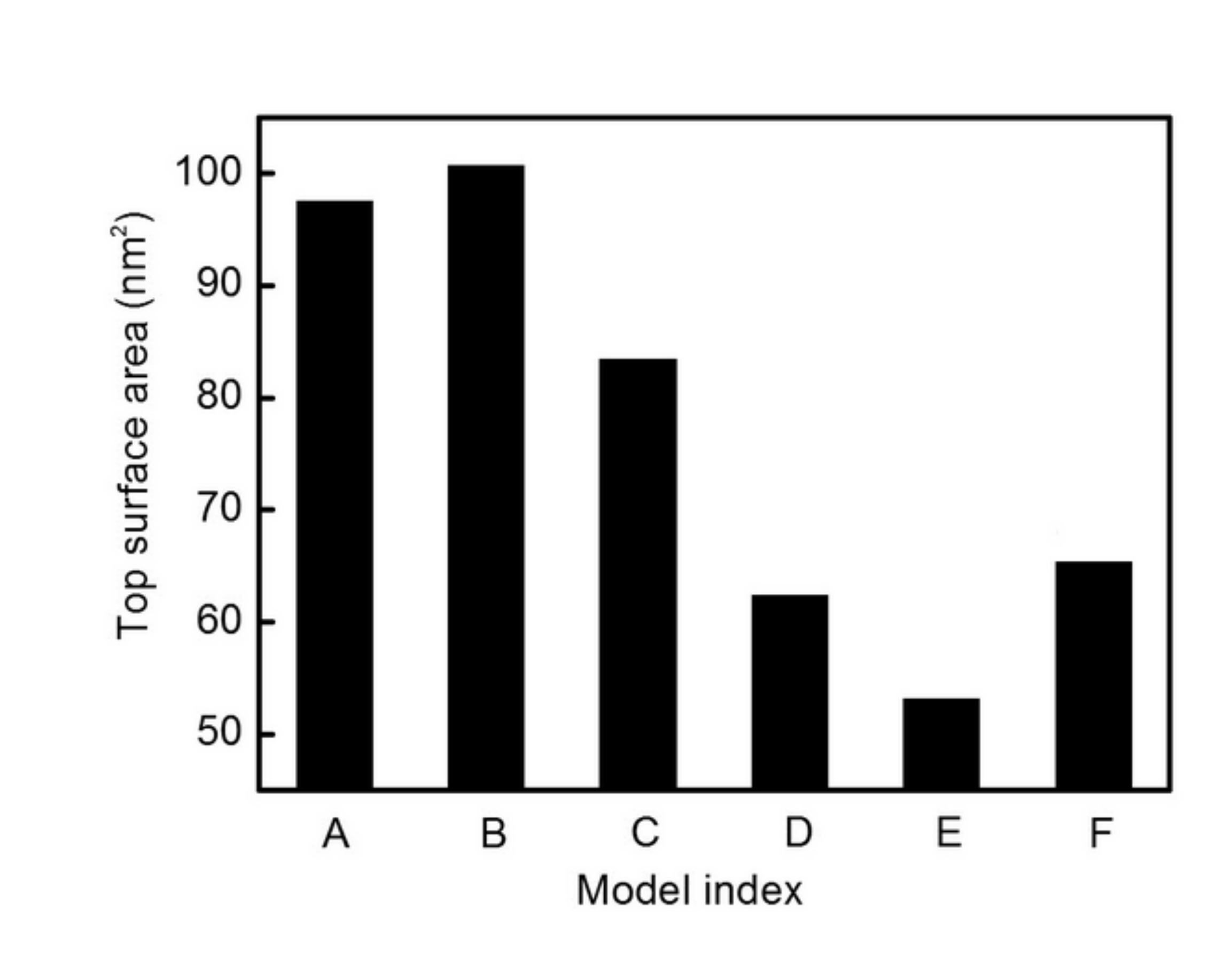

Model index

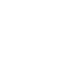

(e)

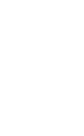

(n)


(a)

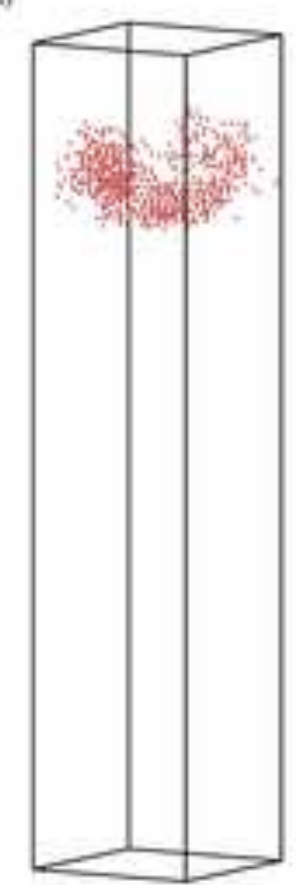

(i)

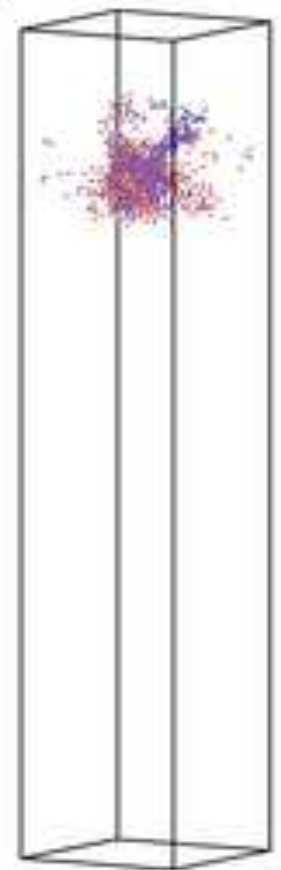

(b)

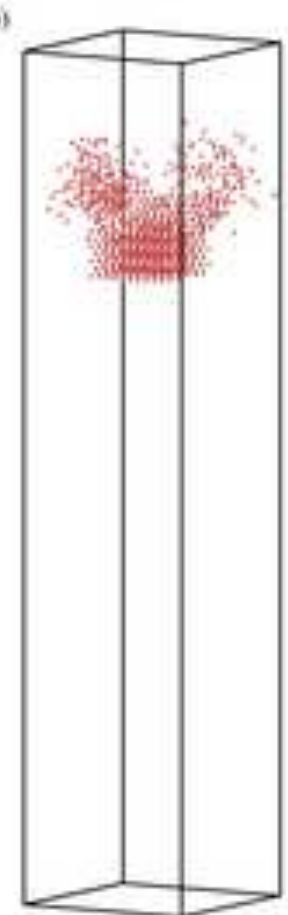

(b)

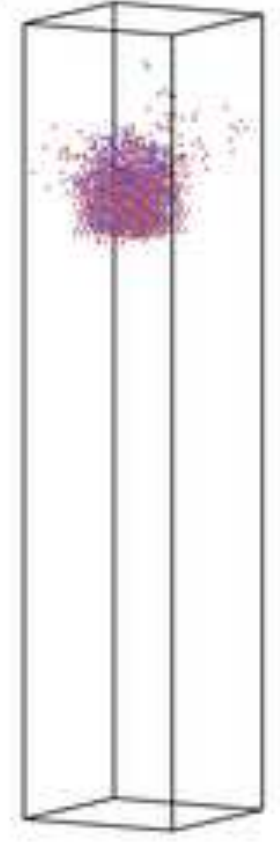

(c)

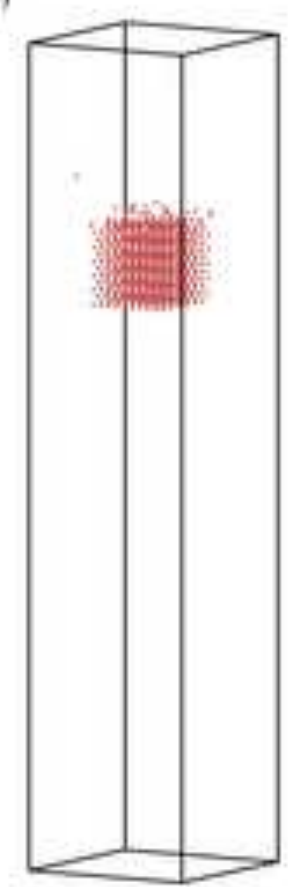

(c)

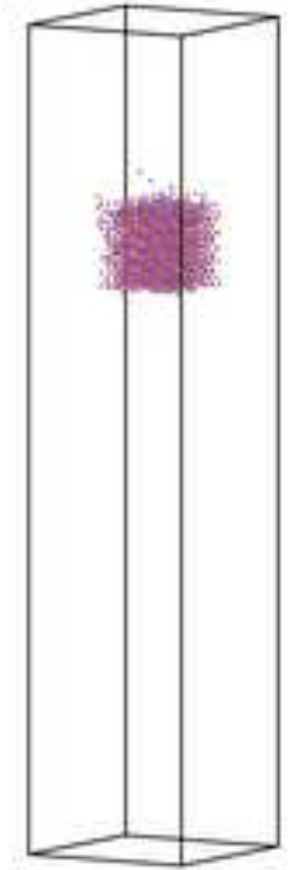

(5)

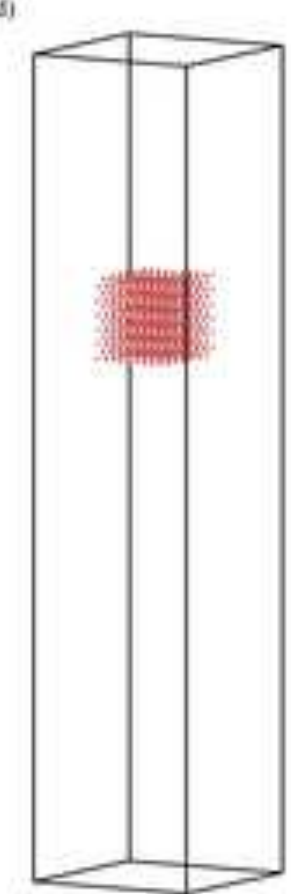

(d)

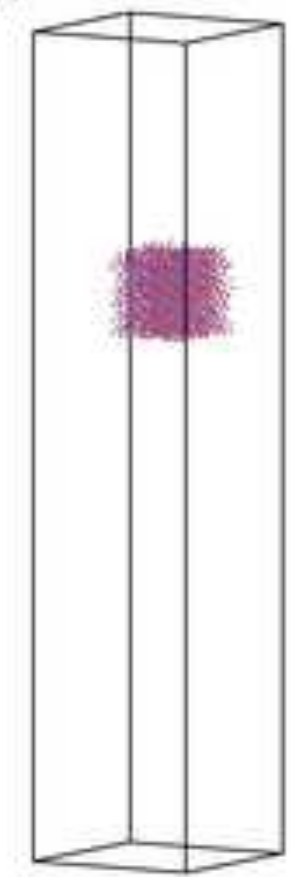

(e)

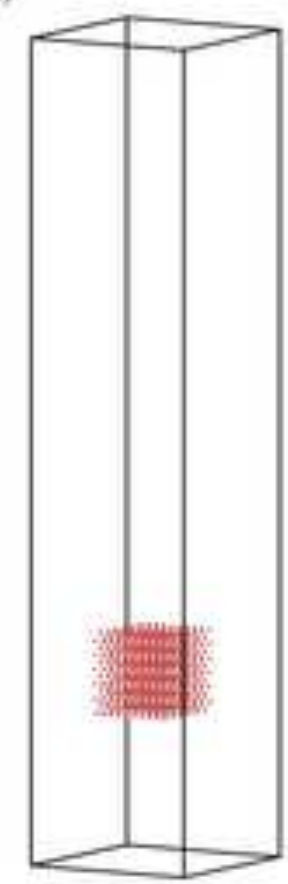

(e)

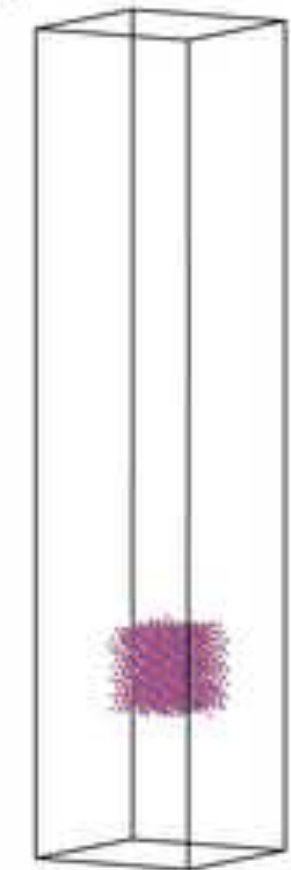




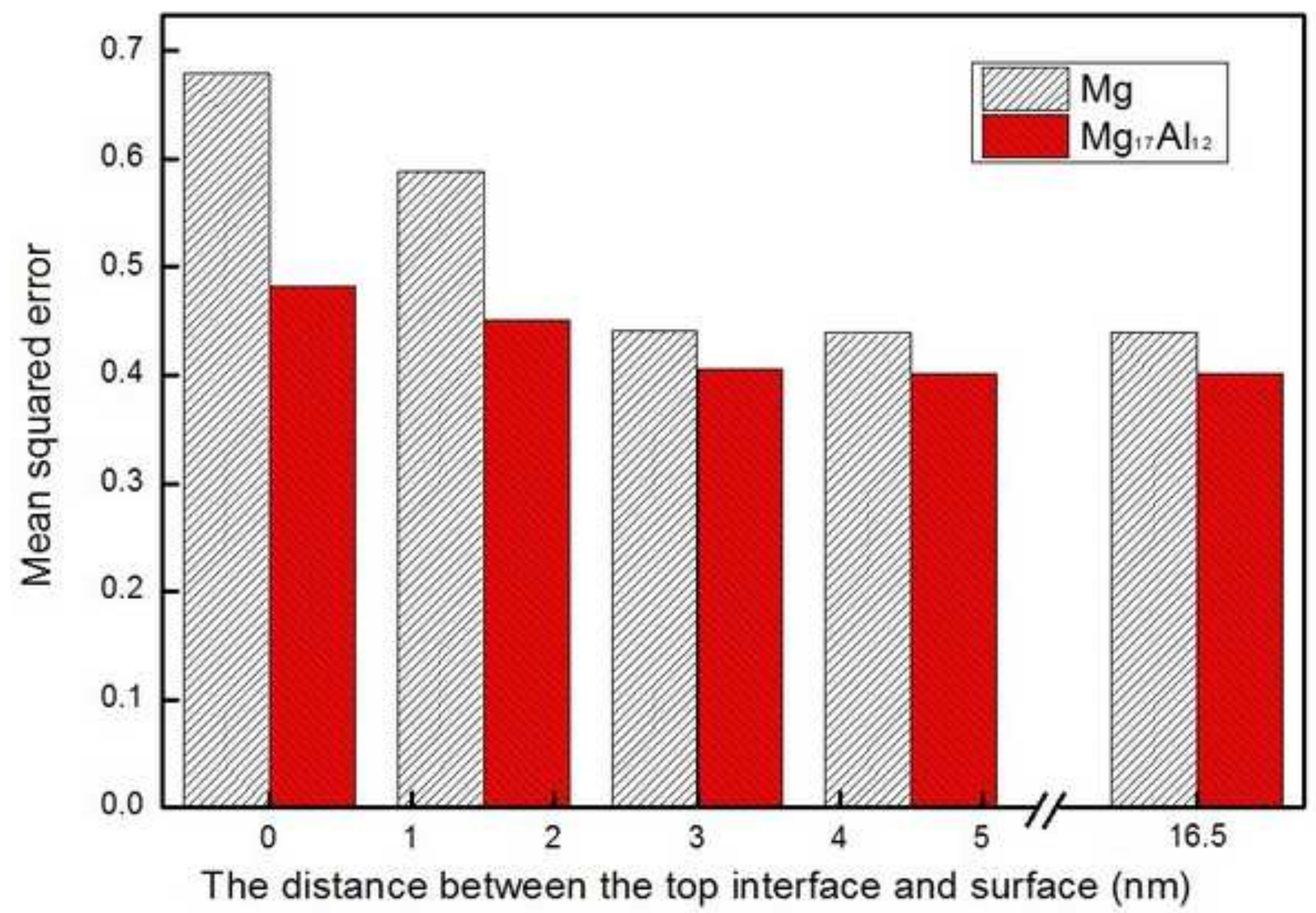

